\title{
Article
}

\section{Transdisciplinary Teaching and Learning in UX Design: A Program Review and AR Case Studies}

\author{
Wei Liu ${ }^{1,2}, * \mathbb{(}$, Kun-Pyo Lee ${ }^{3}$, Colin M. Gray ${ }^{1,2}{ }^{\oplus}$, Austin L. Toombs ${ }^{1,2}$, Kuo-Hsiang Chen ${ }^{4}$ and Larry Leifer ${ }^{5}$ \\ 1 Faculty of Psychology, Beijing Normal University, Beijing 100875, China; gray42@purdue.edu (C.M.G.); \\ toombsa@purdue.edu (A.L.T.) \\ 2 Department of Computer Graphics Technology, Purdue University, West Lafayette, IN 47907, USA \\ 3 School of Design, The Hong Kong Polytechnic University, Hong Kong 999077, China; \\ kunpyo.lee@polyu.edu.hk \\ 4 School of Art and Design, Fuzhou University of International Studies and Trade, Fuzhou 350202, China; \\ kchen@fzfu.edu.cn \\ 5 Department of Mechanical Engineering, Stanford University, Stanford, CA 94305, USA; leifer@stanford.edu \\ * Correspondence: wei.liu@bnu.edu.cn
}

check for updates

Citation: Liu, W.; Lee, K.-P.; Gray, C.M.; Toombs, A.L.; Chen, K.-H.; Leifer, L. Transdisciplinary Teaching and Learning in UX Design: A Program Review and AR Case Studies. Appl. Sci. 2021, 11, 10648. https://doi.org/10.3390/app112210648

Academic Editor: Byung Yong Jeong

Received: 26 October 2021

Accepted: 8 November 2021

Published: 12 November 2021

Publisher's Note: MDPI stays neutral with regard to jurisdictional claims in published maps and institutional affiliations.

Copyright: (c) 2021 by the authors. Licensee MDPI, Basel, Switzerland. This article is an open access article distributed under the terms and conditions of the Creative Commons Attribution (CC BY) license (https:// creativecommons.org/licenses/by/ $4.0 /)$.

\begin{abstract}
Today's user experience (UX) educators and designers can no longer just focus on creating more usable systems, but must also rise to the level of strategists, using design thinking and human-computer interaction (HCI) solutions to improve academic and business outcomes. Both psychological, designer, and engineering approaches are adopted in this study. An invited program review committee met to review progress of the UX program at the Beijing Normal University (BNUX). They considered issues and challenges facing the program today, and the steps that it could make to develop further. During a recent augmented reality (AR) project on designing future life experience on smart home and wearables, several experiential concepts and prototypes were generated to demonstrate HCI and UX research directions. The committee was impressed by BNUX with its energy, enthusiasm, and a sense of purpose on practicing transdisciplinary teaching and learning activities. Recommendations on the current organization of education, the relation between project-based learning and research, and opportunities for exposure and visibility are provided.
\end{abstract}

Keywords: HCI; AR applications; UX design; teaching and learning; NeuroDesign

\section{Introduction}

In recent years, industrial distribution has been changing from manufacturing-centered to service-centered. Studies on user needs have become the basic procedure for product and service feature definition. user experience (UX) has become an essential element that should be engaged in product and service design and has been developed. Companies need talented people with user-centered, innovative, practical skills working with an interdisciplinary and cross-cultural team. The talent demand has pushed the development of UX education. However, the traditional academic research-oriented master's degree can no longer meet the education need for interdisciplinary practical talents in UX. Teaching and learning human-computer interaction (HCI) engineering has primary been concerned with feasibility [1,2], the traditional and technically oriented approach to problem solving. As educators are asked to be more innovative in today's commercial and industrial environment, it becomes critical to weigh in on design thinking [3,4] and design doing [5], transdisciplinary domains [6,7], and global context as well. Pleasurable UX of product service systems is obviously becoming more valued and requires us to focus much more on human values in addition to technical requirements. In recent years, several institutions worldwide have committed to cultivating both innovative research talents and entrepreneurial talents to build a world-class curriculum and pedagogy [8-10]. The huge demand for transdisciplinary teaching and learning in China, and the rapid development 
of information technology (IT), have laid a solid foundation for innovations in multiple domains, e.g., education, healthcare, and personal mobility. With social development and economic growth, the world keeps promoting innovative design and transdisciplinary subjects have become popular [11]. At present, China has a huge number of $\mathrm{HCI}$ engineering educators and students. However, generally speaking, Chinese universities have not set up enough corresponding transdisciplinary education programs for this opportunity, thus well-trained professionals and students are rare.

The development of psychology at the Beijing Normal University has a long history which can be traced back to 1902 . The faculty has first-class research and teaching facilities, and consistently ranks first in the psychology category in China, with comprehensive strengths. Since 2011, universities in China have started to offer professional master's degrees in applied psychology. Beijing Normal University, one of the first universities to offer a master's degree in applied psychology, as the leading academic position in China, provides a solid psychological theoretical foundation and leads the UX education in China with a project-based teaching method under a result-oriented teaching concept. It is a bold attempt to combine UX and applied psychology and it is a reference for other universities offering UX courses.

In 2015, Beijing Normal University (BNUX) was established to train HCI engineers and applied psychology practitioners to keep up with the fast-growing UX economy in China. The program aims to support the design and development of new products, services, and systems in the conceptual design phase by developing innovative methods and techniques, fostering user-centered designers, and leading multi-disciplinary projects. The main research and education directions are innovation and entrepreneurship education, human factors, user research, tangible and embodied interaction design, usability evaluation, and NeuroDesignScience. The program takes in 65 students every academic year. The students come from multi-disciplinary backgrounds, such as (applied) psychology, art and design, mechanical engineering, computer science, information and communication engineering, education, business management, international trade, statistics, logic theory, etc. BNUX is aware of the key issues that are fundamental in education: (1) UX is now acutely different given the arrival of $\mathrm{HCI}$, machine learning, and artificial intelligence (AI); (2) awareness that context is a critical variable and a challenge to quantify; (3) deep awareness that quantitative and qualitative research is needed to reveal the underlying variables; (4) design thinking is a curiously neglected intellectual activity in the context of science thinking; (5) it is critical that we develop a balanced approach to our social networks through balanced attention to the design and science paradigms. BNUX offers strategies for the transdisciplinary teaching and learning challenges our cultures now face. It produces UX designers, but more importantly, it gives students the tools they need to become better humans and to change the world for the better.

\section{Approach}

The teaching focuses on project-based practice, which is the outcome of the course, and the cultivation of students' practical abilities. The teaching team integrates psychology resources from the mother faculty with industry project resources to provide theoretical knowledge and practical conditions to ensure learning outputs and encourage students to participate in social practice activities, such as product transformation or competitions. The UX program is committed to integrating psychological knowledge and practice skills in the real world to cultivate applied talents. At present, UX significantly values design education that is carried out from the human-centered perspective, but it often lacks the support from basic psychological theory. The demand for graduates' ability in the industry is no longer satisfied by the universal ability of interface design or interaction design but needs more psychological theory to engage in user research and analysis. The core value is to research people and their cognition and behavior.

The curriculum provides a sufficient academic and theoretical basis for practical application. At the same time, the curriculum promotes the transformation of psychological 
theory to application and balances the relationship between academic research and practical application. The courses are composed of four modules: psychology, design, HCI technology, and business, which form an interdisciplinary system. (1) The psychological module strengthens the students' theoretical knowledge of psychology. Teachers focus on the explanation of theoretical application and the analysis of practical cases. (2) The design module enables students to have solid practical skills. These courses are mainly project-based, providing students with a natural project training environment. (3) The HCI technology module includes the application and product development on virtual reality (VR) and augmented reality (AR), and intelligent hardware trains for prototype making and iteration, and further expands students' mastery of product development and process knowledge. (4) The business module mainly cultivates students' market and business operation ability to understand the classic business model and the latest business model innovation in the market. This curriculum enables students to foster a user-centered way of thinking based on psychology and teaches professional skills such as user research, product planning, usability testing, information architecture, interaction design, interface design, business model innovation, etc. The application of these practical skills is intertwined in various courses. Through constantly repeated training, students deepen their application of theory and understanding of methods and processes and improve their practical ability to cultivate applied talents.

\subsection{Human-Centered Design (HCD)}

$\mathrm{HCD}$ requires us to collaborate with and understand more fully the approach of social scientists in the cognitive psychology, sociology, and cultural anthropology fields [12,13]. Our challenge and opportunity after developing expertise in the social sciences is to understand human values, and this needs to be near to the same extent that we understand technical and analytical issues. This allows us to design products, services, and experiences that people truly value as individuals and as a culture. Instead of inspiration coming primarily from new technical advancements that we are trying to exploit, we take the approach of studying and observing humans to understand their wants and latent needs and to design appropriately satisfying solutions that make a difference. BNUX houses the disciplines of human factors engineering and design science. The emphasis of both disciplines is on the interaction between human and products. Key aspects are the expressiveness of products, usability, and aesthetics; the meanings that product forms have for their users; and the place of design and designing in a socio-cultural context. The program incorporates the above elements into both research and education. BNUX focuses on user, context, emotion, interaction, technology, and human factors through practicing innovation design thinking.

\subsection{Engaging Transdisciplinary Teaching and Learning}

While many traditional design disciplines still draw heavily on studio-based traditions and themes of disciplinary knowledge that have roots back to the Bauhaus or École des Beaux-Arts, efforts to define inter- and transdisciplinary engagement have been the focus of many emerging design disciplines. The introduction of new approaches is needed to support innovative practices but brings with it the complexity of selecting among multiple sources of disciplinary knowledge and traditions, and then building alignment across program staff. The Design Enterprise Model from Faiola [14] proposed integration of knowledge domains that included social (human and culture), design (graphic and interaction), business (market value and return on investment), and computing (building and testing), with attention to integration across these domains through theory, application, and management. This model mirrors contemporary transdisciplinary engagement in UX design practice, with connections across multiple domains of disciplinary knowledge, such as psychology, computer science, anthropology, cognitive science, design, and ethics. This multiplicity of knowledge domains points towards complexity relating the epistemological challenges of forming coherent educational experiences, and complexity relating to the 
identity construction work students need to undertake to effectively work within and across disciplines $[15,16]$.

\subsection{Student Teams}

Students come from different backgrounds and disciplines, e.g., psychology, industrial design, $\mathrm{HCI}$, and economics. The diversity assures that teams take multiple perspectives on the given design briefs, increasing the probability of breakthrough discoveries and innovation. All students have core competencies in their respective fields, and many have prior design project and HCI research experience in academia or industry. Unlike many other academic psychology projects, which require students to optimize one variable, students must design an experiential AR and NeuroDesign system while being mindful of not only the primary feature but also the usability, desirability, and societal implications. BNUX trains students' comprehensive abilities through coursework and practice in workshops, including user research, product research, design innovation, technology integration, reflection, criticism, leadership, collaboration, visual and interactive expression skills, and international perspectives. Students apply these skills to solve real-world problems. They complete tasks and gain skills and experiences at the same time. Each team visits the corporate partner regularly for reviews and presentations. This learning experience improves students' communication and professional skills.

\subsection{Corporate Partners}

Companies, small and large, from immersive $\mathrm{HCI}$ industries are invited to bring forward their innovation design briefs. The BNUX researchers consult with corporate liaisons to define the right scope and scale of a project. Liaisons are recommended to keep in regular contact with the design teams to provide feedback. The student teams are assigned industry supervisors who are the stakeholders in the companies and working in a field related to the design briefs. They provide a great resource to the student teams who can access a wealth of knowledge through the supervisors and their social network. In the organization of teaching, teachers transform the position from professors to organizers and coordinators, coordinating all resources. In the process of teaching, teachers and industry supervisors develop teaching plans and methods together to ensure that needs are real, methods are practical, and solutions are down-to-earth. This provides the student teams with sufficient practical opportunities that are in line with the industry. After being employed, students can quickly adapt to the working environment and rhythm, which shortens training time.

\subsection{AR and Experiential Prototyping}

Prototyping is at the very heart of the design process because it is the most effective way to transform ideas into tangible products [17]. Students create numerous prototypes to articulate their vision and test their design assumptions. Through iterative prototyping in many ways, broad problem statements are refined into concrete concepts that are eventually incorporated into a fully functional prototype $[18,19]$. AR overlays digital content and information onto the physical world, as if they are actually there in the users' own space. AR opens up new ways for the Internet of Things (IoT) devices to be helpful by letting the users experience digital content in the same way they experience the world. It lets the users search things visually, simply by pointing cameras at them [20]. It can put answers right where questions are by overlaying visual, immersive content on top of the real world. For example, the users can use AR to place 3D digital objects right in their own space directly from Search or from websites on Chrome, and with Live View in Google Maps, they can quickly orient themselves and know which way to go with directions overlaid right on top of the world. In VR and AR design and development workshops, multidisciplinary knowledge is integrated through interdisciplinary team collaboration. The application of psychological theory enables the student teams to define motivations behind design 
principles and create HCI solutions that satisfy people's perception, attention, cognitive ability, and emotion.

\subsection{The Applied Psychology Research Labs}

Across over 20 applied psychology research labs at the Beijing Normal University, a human-machine interaction (HMI) lab was designed to train psychology students and junior $\mathrm{HCI}$ engineers to conduct experiments on future ways of teaching, learning, transporting, and playing. We specifically investigate HMI for creating new AR, virtual reality (VR), and mixed reality (MR) experiences and experiential prototyping solutions; and test the usability by analyzing data collected from eye-tracking, electroencephalograms (EEG), electromyograms (EMG), and functional near-infrared brain spectroscopy (fNIRS) devices.

\subsection{NeuroDesign}

Based on the research by Reiss, Saggar, and others [21-23], a new research track called the Leifer NeuroDesign Research Program (neurodesign.stanford.edu, accessed on 7 October 2021) at the Stanford University emerged. Other universities have joined, including the University of Potsdam, the Beijing Normal University, and the Tokyo Institute of Technology. This intersection between psychology and design has since evolved into design thinking, a practical approach to innovation. The program aims to investigate thinking in design, team performance, and practices through approaches from $\mathrm{HCI}$, design research, experimental psychology, and neuroscientific instruments. It aims to examine team practices that produce meaningful, innovative, and practical design [24,25]. Our research investigates design activities and related thinking from various perspectives, including neuroscience, embodied cognition, Gestalt, and other phenomenological perspectives.

\subsection{Reflecting and Validating the Approach}

The recent emergence of new undergraduate and graduate design programs with a focus specific to UX offers new opportunities to engage with the complexity of these educational $\mathrm{HCI}$ practices. In a series of twenty interviews with students and faculty to describe transdisciplinary teaching and learning between BNUX and the UX Design program at the Purdue University, the research team reflected on how each program was created, developed, and iterated upon, describing program goals and student experiences across both programs from student and instructor perspectives. This approach implies the need to identify opportunities for educational partnerships that enrich student experiences and future industry success that are culturally and locally bound while also recognizing the role of subverting or extending institutional support and structures. This finding points toward the need for future work that engages with the experiential qualities of $\mathrm{HCI}$ and UX curriculum, representing a shift from past approaches that have primarily focused on objective and visible elements of education. More research should identify the implicit norms of existing curriculums, using action-oriented research methodologies to propose new student experiences in UX-focused programs. The research team identifies a range of factors that influence the formation and performance of studio culture in these environments, including instances where culture, social, and contextual factors constrain or enable particular kinds of educational experiences. The team concludes with opportunities for future research on UX and HCI educational practices, including a greater focus on local educational practices, VR and AR HCI, and a multiplicity of curricular philosophies.

\section{Discussion in the Program Review}

The curriculum structure is divided into three levels: (1) The first level is the psychology theory courses, which train students to obtain rigorous research thinking and solid psychology experimental design methods. The students need to understand physiological characteristics and limits of human beings when they ideate HCI solutions. (2) The second level is the UX foundation courses, which enable the students to master basic UX design process and conduct user research through qualitative and quantitative methods. (3) The 
third level is the UX professional skills courses, a set of career-oriented courses designed to broaden students' employability, including interface design, product development, and service design. These courses are all professional elective courses, which provide a platform for students to deepen their study in a particular field. At the same time, the program offers extensive extra-curricular workshops and lectures to expand students' comprehensive capabilities. To enrich the overall curriculum, renowned experts and scholars from the industry and academia are invited to give workshops and lectures on cutting-edge $\mathrm{HCI}$ research to broaden students' horizons on current trends related to UX. This curriculum structure provides students with a solid foundation in psychology and achieves practical training for applied talents through project-based learning.

BNUX is unique in China, possibly in the world, as a UX and HCI program located in a psychology faculty. Most other similar programs are located in arts, design, or engineering schools. This has both drawbacks and benefits. Drawbacks include 'having to survive in a psychology faculty', with a culture of journal publications and quantitative scientific method [26]. Just as in design and engineering schools, harvesting scientific articles from project work is a challenge. Many beginning writers tend to report on what was done, rather than which findings were found. Learning to write from the viewpoint of the intended reader, i.e., identify the audience associated with the journal of conference, is important to frame the work in the questions and references of that community [27]. In other words, when carrying out user-centered design for academic writing and communication, identify the user, the user's needs, and explain the contribution in terms of those interests. Regarding these, it would help to make use of the new international developments in research for several reasons:

- Generating attention for societal impact, as shown by companies and others making use of BNUX results. There are some examples of exercises, e.g., the Delft research assessment self-report lists some possible indicators other than the social sciences citation index (SSCI) journals and attempts to define how it relates to 'important journals in the field'. However, BNUX should make its own map of important journals, conferences, and benchmark institutes for UX and HCI. To convince psychology colleagues that 'quality is not necessarily three completed experiments', BNUX must have a strong story (with examples) about what other aspects are important, and why. BNUX may try to find birds of the same feather around the world and explain that we are not an outlier. If we are not explicit, all they hear will be 'we want to do less', which is not what we mean.

- Using the academic discussion on knowledge forms other than 'high theory', such as mid-level knowledge [28-30], and emerging formats, such as the pictorials at the association for computing machinery (ACM) designing interactive systems (DIS) conference (dl.acm.org/conference/dis; accessed on 5 October 2021). These give academic credibility to HCI design results.

- Part of the UX output is in design suggestions and prototypes, just as for regular 'design'. Have some form of exhibitions to show these. It would be very valuable if we can find a format in which (some of) the psychology colleagues also collaborate, so they also have ownership, or are 'partners in our success'. It may be important to devote attention to the aesthetic qualities of prototypes. A functional Arduino-based prototype may convince the insider of a design proposal, but for an outsider it has to look 'real' to be convincing. On the latter, the aesthetic qualities require getting closer to cultural aspects, not only local ones but also the study of societal dynamics and phenomena that might be happen in the world, i.e., international trends, demands from the market, and socio-cultural influences.

- Finding examples of how other design and HCI institutes are dealing with publishing in psychology conferences and journals. Where are the psychology communities that are already convinced that design can have an impact in their field? The psychology strength could show both in the content (e.g., naming theories and principles that are 
used from psychology literature) and format (e.g., presenting the quantitative analysis of statistical data with the appropriate statistical measures).

- Not leaving it up to the student teams to connect the psychology and UX and HCI courses. First, make sure we know what happens in both so in the courses we can guide students in understanding the connection. Next, develop co-creative activities with the psychology professors to help them bridge the gap from their side. For instance, a workshop with some psychology professors when HCI and design psychology experts (e.g., Prof. Pieter Desmet and Dr. Atsushi Maki) are at the faculty.

\section{AR Case Studies}

To take lessons learned from the program review and to explore future ways of transdisciplinary teaching and learning supported by emerging AR technologies, a recent project entitled 'designing future life experience on smart home and wearables' was conducted with one of our corporate partners. This project was aimed to provide the AR research team with first-hand user insights, design ideas, and mockups on new ways of immersive interacting and ambient computing for future smart hardware opportunities towards smart home and wearables in a Chinese context. The research questions were as follows: What does wearable mean in China? Why? What are typical scenarios and user groups interacting with the wearables? What are the AR and UI design opportunities? Several conceptual designs and developments were generated. Examples are described below.

\subsection{More Than a Cane}

There are about 2.2 billion visually impaired people globally, while there are more than 17 million of them in China, which means that there is one visually impaired person in every one hundred people. Although many people already know that guide dogs can be the 'eyes' of the visually impaired, they also have something challenging to identify, as the guide dogs cannot identify the color of traffic lights. With intelligent image recognition technology development, open-source algorithms can help the visually impaired solve these problems. The form-giving was inspired by whales as they live in a dark and lonely environment in the deep ocean.

A scenario is as follows. Kang participates in an activity in a community club and is given a multi-scene intelligent cane with account points: More Than a Cane, a product to solve the difficulties of crossing roads and daily information recognition visual impairment. When Kang arrives at the traffic intersection, he turns on the camera to obtain image information. The cane judges the color of traffic lights through an intelligent hardware algorithm, enables sidewalk green light recognition, and feeds back through a vibration sensor. Kang crosses the road smoothly after feeling the vibration and hearing a voice prompt simultaneously. At home, he stows the cane body and sends a drug manual's text information through the camera of the handle. After text recognition, the cane reads out the content of the drug manual and helps him understand the dose method. The prototype and part of the coding are shown in Figure 1. The form-giving design is adjustable and can be applied to multiple scenes. The specific HCI logic diagrams are shown in Figure 2.

In terms of hardware engineering design, the cane consists of five main components (Figure 3): (1) a Raspberry Pi camera module V2 for taking photos, (2) a Gravity's digital touch switch for controlling the camera (i.e., turn on and off), (3) a Gravity's micro-vibration module and a Fermion's DF1201S DFPlayer PRO MP3 player module, (4) a core processing module of a Raspberry Pi ZERO with integrated WI-FI and Bluetooth, and (5) a power module of 18,650 Li-ion battery charger and wireless charging base and chip. The hardware design is as follows: AI cloud computing is the core technology, using Vision API and ICDAR to recognize the traffic lights and texts. Through testing in the real context of streets and shops, the research team discovered that the background information seriously interferes with the recognition of the traffic lights. Therefore, an independent code was built in the cloud to recognize information in two dimensions: colors and graphics. After five iterations of form-giving design and taking and testing more than 1000 photos, the 
recognition accuracy was $94 \%$ and the recognizable distance increased from three meters to fifteen meters. Furthermore, the cloud computing and machine learning enabled AI evolvement of the experiential prototype itself over time and context of use.
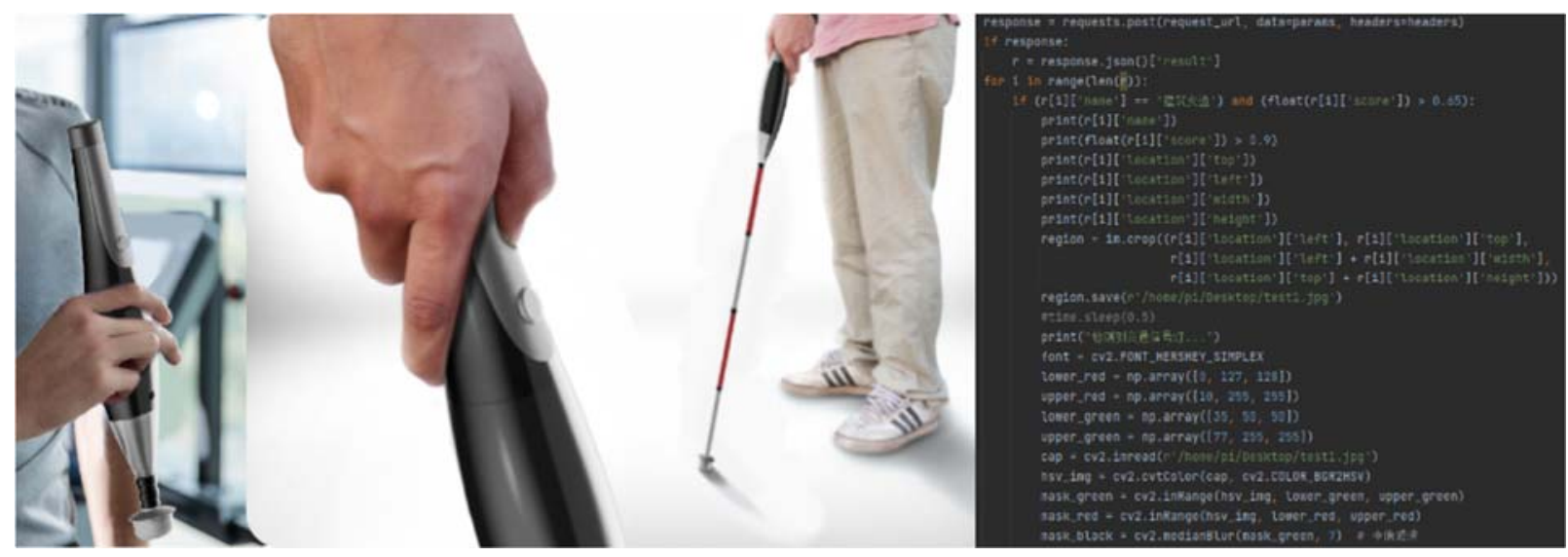

Figure 1. The logic diagrams of identifying the traffic lights and the text content.

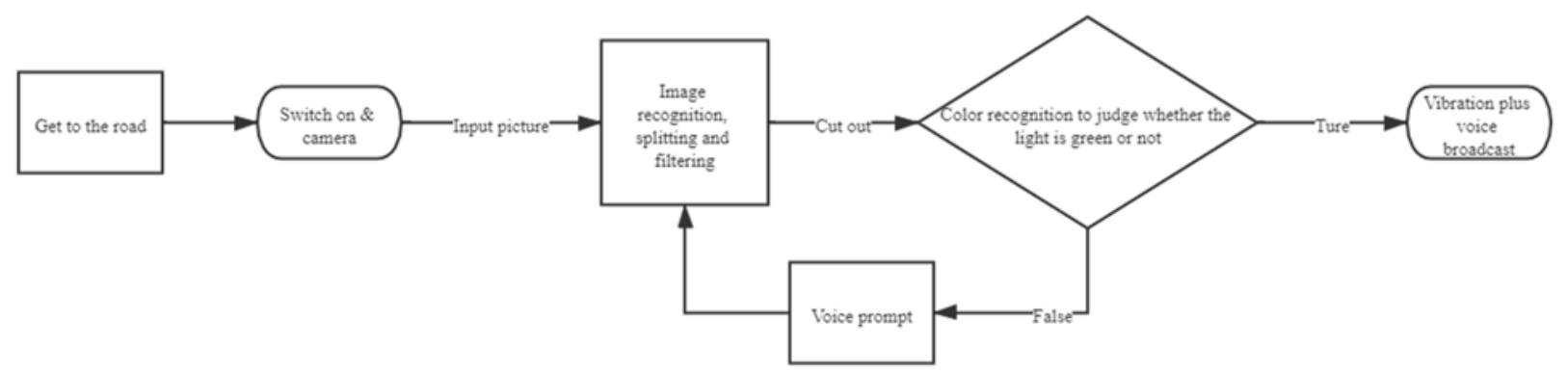

Figure 2. The HCI logic diagrams of identifying the traffic lights and the text content.

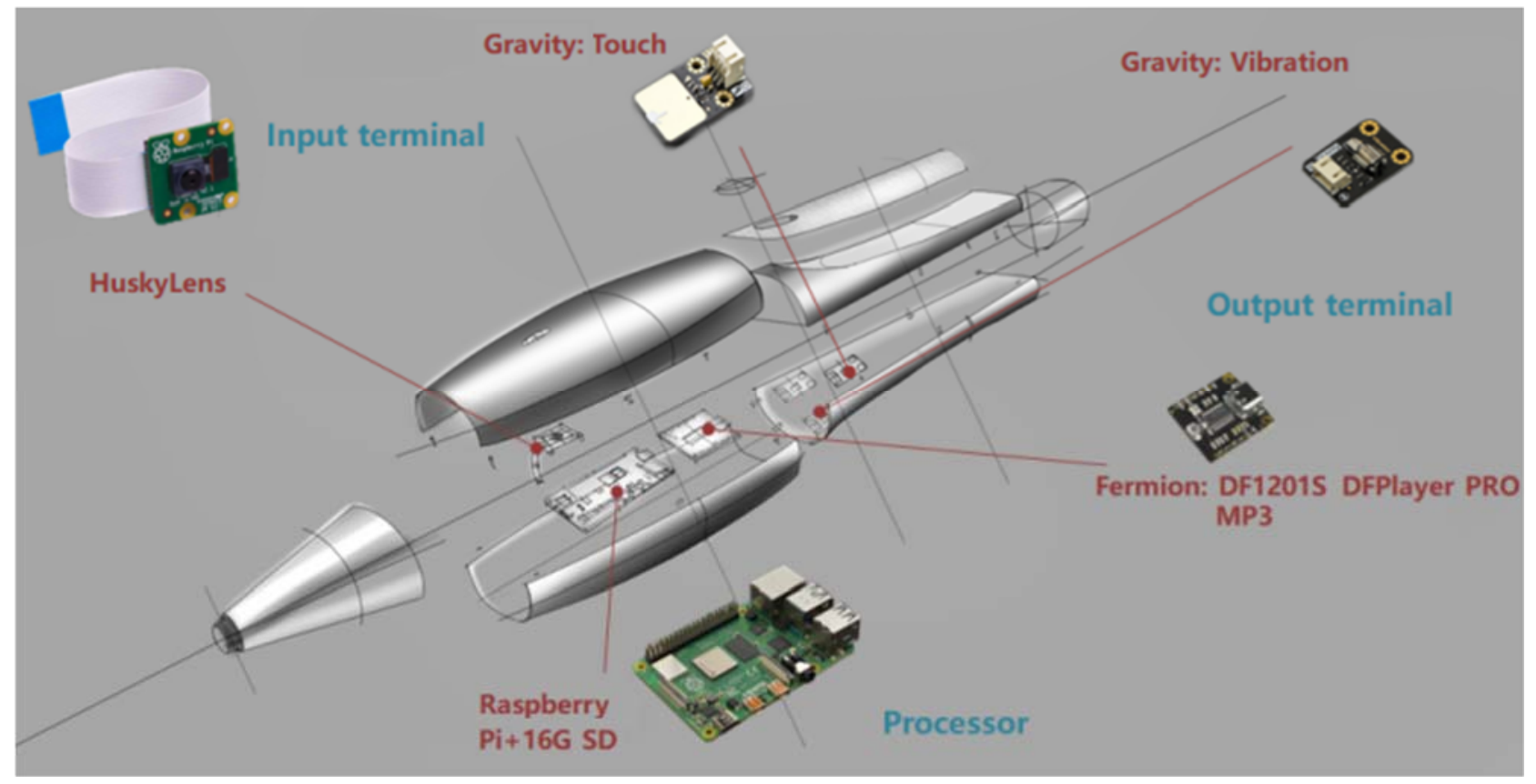

Figure 3. The five main components of the hardware engineering design. 


\subsection{The Go-Light}

Parents who have kids between three and six years old have some safety concerns at home when they must leave temporarily. Currently, they are trying to solve this problem by simple measures, for example, giving more talk and attention to their children, but their current solution only solves some of their problems. To solve this problem, the student team designed a smart AR lighting and matching mobile app. The safety data is delivered to users in the app. A successful design is time enough to prevent children from danger and flexible enough to adjust danger points at home according to the degree of danger.

The scenario is as follows (Figure 4): As a working mother, Qin receives an urgent notice from work. She rushes to have a video conference and leaves her four-year-old child, Cong-Cong, playing alone in the living room. After playing with toys for a while, he feels boring and explores around. He finds a pack of colored pens and is excited to have them. The position of the pack is relatively high on a shelf, so he cannot see a pack of pushpins by the colored pens. When his hand approaches at a dangerously close distance, a smart light from the Go-Light detects the potential danger and projects a smart AR elf in front of Cong-Cong to block him. A voice prompts (his mother's voice) 'Cong-Cong, don't touch this, there are pushpins, and they will stab you'. He puts down his hand and goes back to read his picture books. At the same time, the smart light detects that the blocking is successful and sends a short message to the mother's mobile phone to explain the situation. After the video conference, Qin lets the smart light cast on the wall and reviews what happened. She is delighted with the smart light.

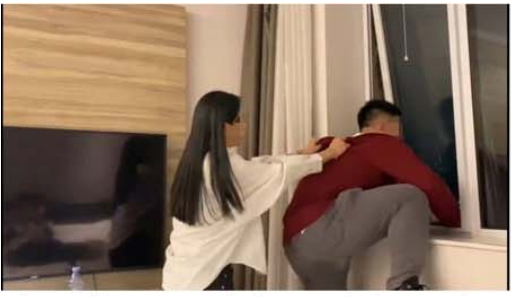

The child tries to climb out of the window, and mom stops him immediately.

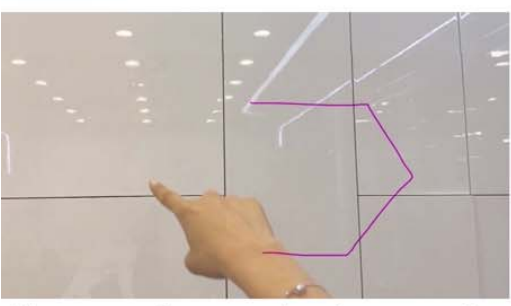

The mom performs a gesture to summon the Golight elf.

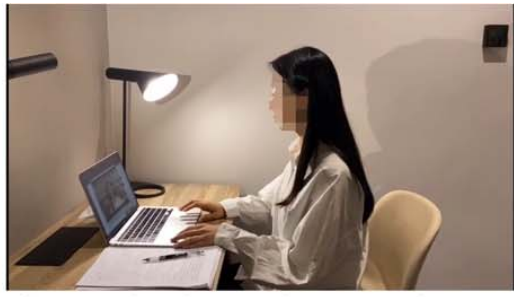

The mom does her own business and cannot keep an eye on him all the time.

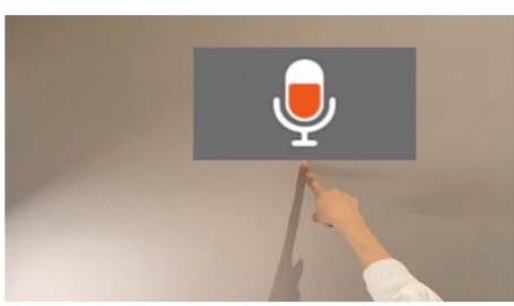

The mom presses and holds for voice chatting with the child.

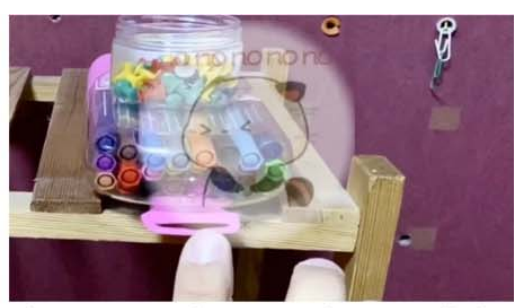

The projection of a cartoon character tells the child to stop dangerous behaviors.

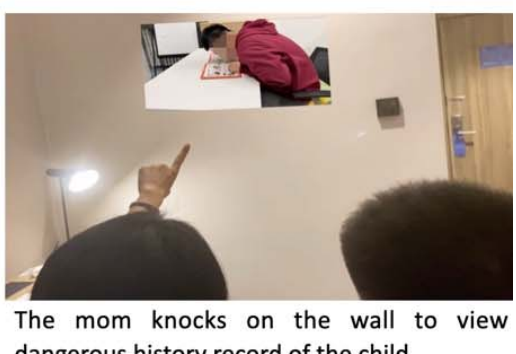

dangerous history record of the child.

Figure 4. The scenario of the usage of the Go-Light.

The smart light and mobile app establish a linkage, and through the smart light comprehensive scanning room the user sees a danger level on the mobile app and can prepare corresponding protection measures in advance. There are several gesture interactions [31]: draw a hexagon (the shape of smart lights) to turn on the safety elf, press and hold to enter voice, tap to review danger history, draw the hexagon in the opposite direction to turn off the elf, etc. (Figure 5). The features are demonstrated and realized through AR prototyping (Figure 6). According to characteristics of different rooms in the home, different shapes and personalities are given for the elf, such as a rigorous elf for a study context. The elf presents a three-dimensional image and is able to dance, jump, spin, and move around with support from the corporate partner's AR research platform. This can increase childrens' sense of substitution and make children more easily to accept the advice. In addition, the smart light can build game scenes through AR and teach safety knowledge through these games. 


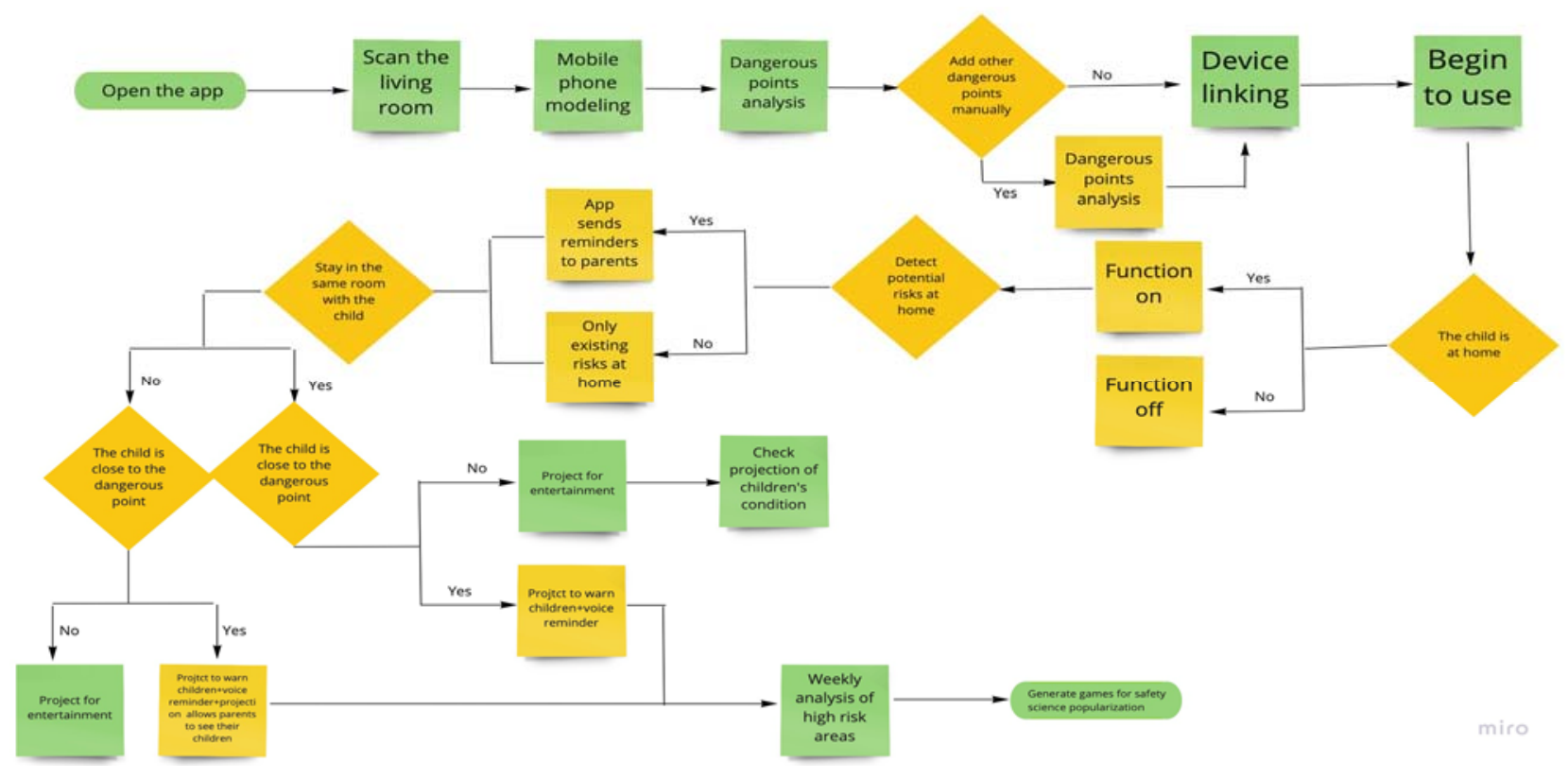

Figure 5. The HCI logic diagram.
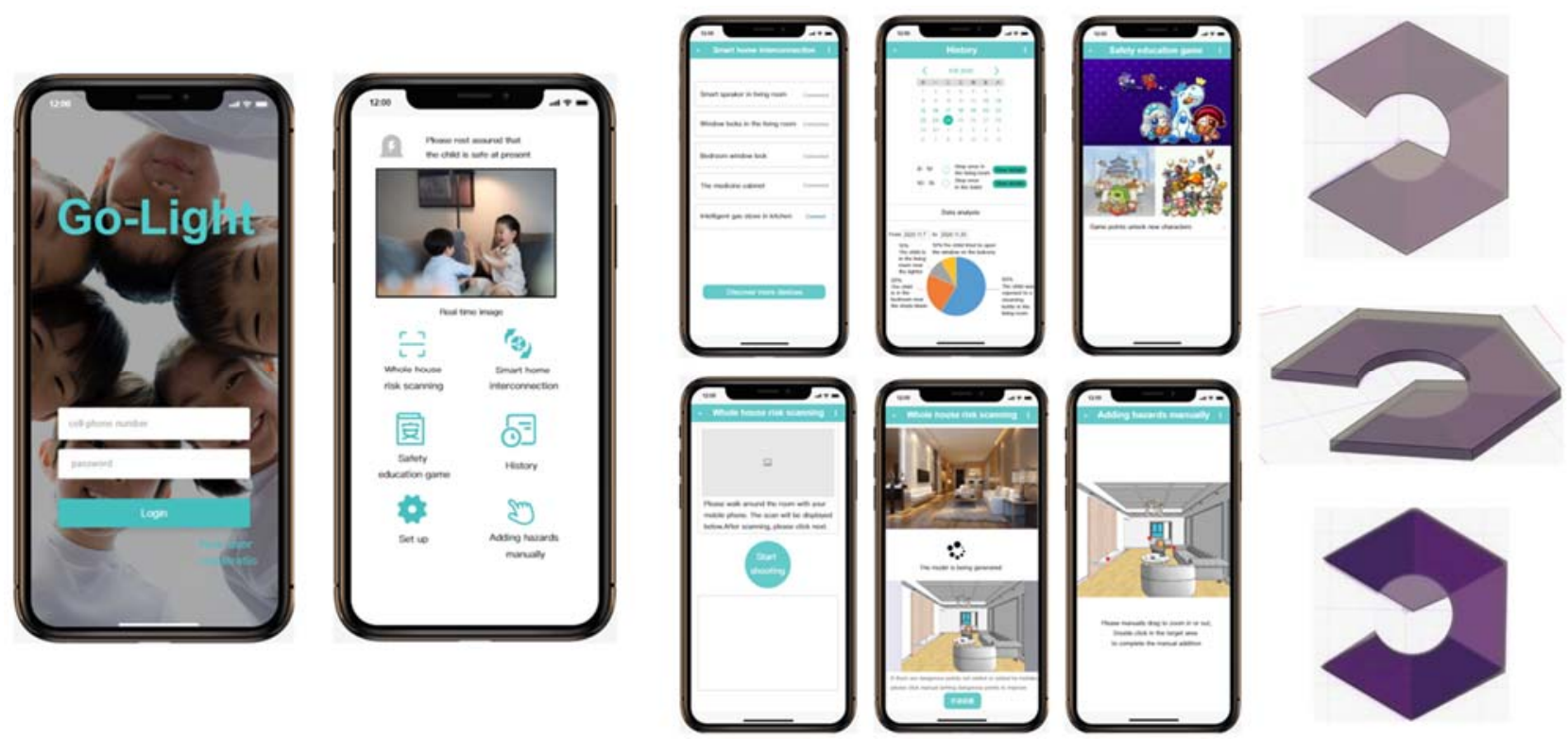

Figure 6. The user interface prototypes of the mobile app. Different shapes and personalities are given for the elf according to different home contexts.

\subsection{The Cube}

Dama (which are generally a group of women deeply influenced by Chinese tradition and are willing to serve the household and are concerned about daily expenses) have difficulties in keeping up with the rhythm when finding a song that is suitable for square dancing. Currently, they have tried to solve this problem by practicing for a long time or reluctantly switching to another song, but their current solution is time-consuming, leaves them feeling unhappy, and is inefficient. To solve this problem, the student team designed an immersive dance service to be delivered to users on accessories. A successful design is relaxed, easy to use, enjoyable, natural, and collaborative for dancing and learning.

The scenario is as follows: Aunt Jiang has been retired for two years. Recently, she started dancing on the square in her community. However, there are many people, she 
can only stand in the last row, and still has to learn to dance. She cannot keep up with the rhythm and catch the coach's movements. She is frustrated. In the evening, she comes to the square early after dinner, brings the Cube, and wears it on her waist as a belt. Aunt Jiang pats Aunt Zhang's shoulder, and their belts generate voice cues 'the team is matched'. The dance starts and she starts to panic again. The Cube projects a signal light on the ground in front of her and makes buzzing vibration on the waist to indicate that she should keep moving in this position for a few seconds. With the weakening of the vibration, she understands the next and repeating movements. She feels much more confident than before. When she returns home and unlocks her mobile phone, her belt automatically generates and uploads data and visual charts of the dance performance. 'Today, my dance performance even ranked top 30 in my community', Aunt Jiang shouts. Besides dancing, she worries about not being able to find suitable songs for dancing. Even if she finds a fitting song, she does not know how to download it, so she has to ask her children to help out every time. The Cube helps her by simply clicking a floating button on music streaming to download and store directly on her local devices. She makes a grab gesture in the air to play a song and makes a flip gesture in the air to switch songs.

The interactive interface is projected in the air when wearing the belt. The interactions are through gesture recognition (Figure 7). The user chooses different dance modes and different songs. When the dance starts, the belt reminds the corresponding dance posture through vibrations and lights. By tapping friends who are also wearing the belt, they dance in a group and share the same songs and beats. With support from the corporate partner's AR research platform, the prototype applies a hardware base of high-precision radar that enables the recognition of fingers in a micro-interactive way. This hardware sufficiently supports the gesture recognition. The acceleration sensor judges the user's movement and matches the lighting and sound effects.
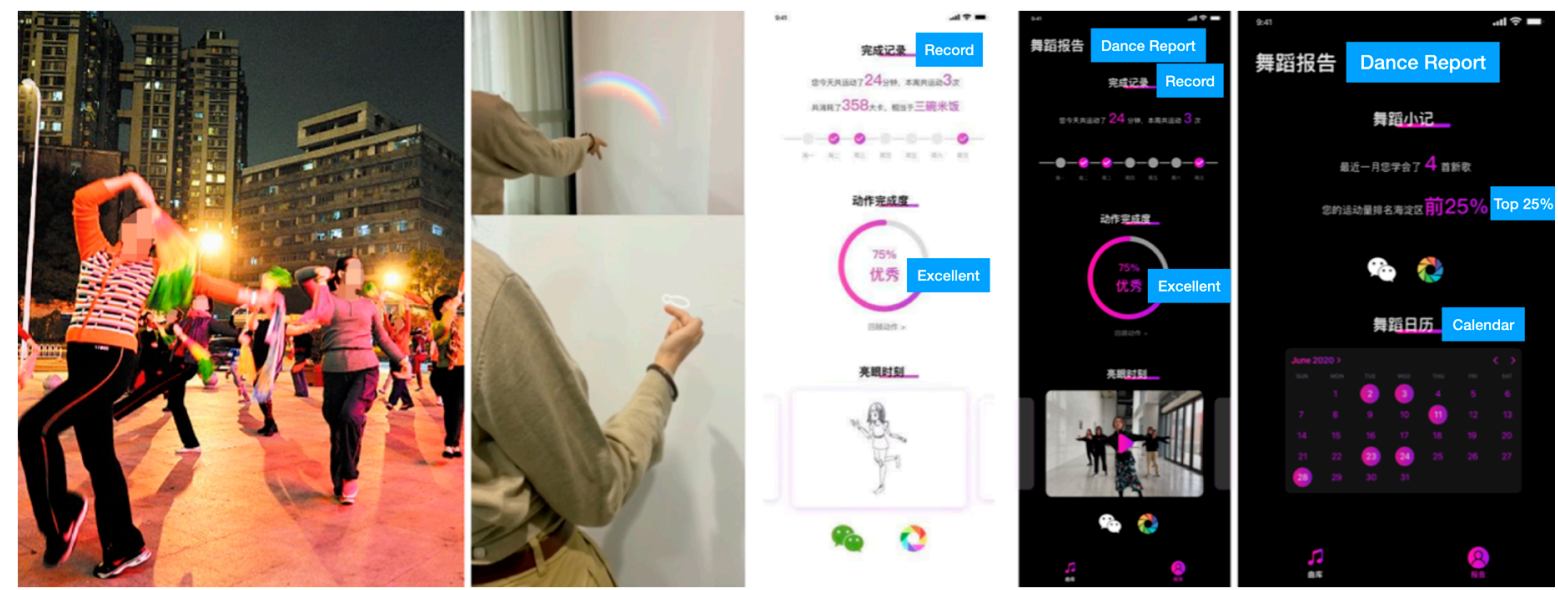

Figure 7. The gesture interactions and user interface prototypes of the mobile app.

\subsection{The Toast!}

Restaurant experiences often result in uncomfortable, or even non-existent, communication among young adults and parents. Young adults wish to use their cell phones and continue engaging with friends online, while their parents want to engage their child in verbal communication. To address this problem space, students in the Tangible Embodied Interaction module, co-taught by authors Gray and Toombs, used an AR space, along with a few IoT devices, to enhance the restaurant experience to support and facilitate conversation.

In this experience, the student team leveraged various physical aspects of the restaurant experience to make the interaction feel natural, engaging with principles of proxemic interaction [32] and ubiquitous computing [33] that they had learned about in the module. The team used the bodystorming method approach to role play the experience through 
multiple iterations of the design, resulting in a polished set of interactions, some supported by an Arduino prototype, in their final presentation (Figure 8). First, they added sensing capabilities to the water glass, using the glass as a way to promote a conversation without the need for phones or awkward silence. The cups could be tapped to display a conversation-starting question and 'toasted' with that of a family member to proceed to the next conversation starter. Secondly, the chopsticks gained a new sensing capability and were designed to enable hand-written responses to conversation starters, which would then be incorporated into a multiple-choice trivia game, enabled by a display on the dining table. These technological augmentations retain the typical aspects of a dining experience but reimagine what that space might feel like for family members without a smartphone at the center. The playful and collaborative interactions afforded by the conversation starters and continuing engagement with family members create the potential for a restaurant experience beyond smartphones. However, perhaps it is optimistic that only having a question on one's cup would help people open up or relearn how to communicate verbally with family members.

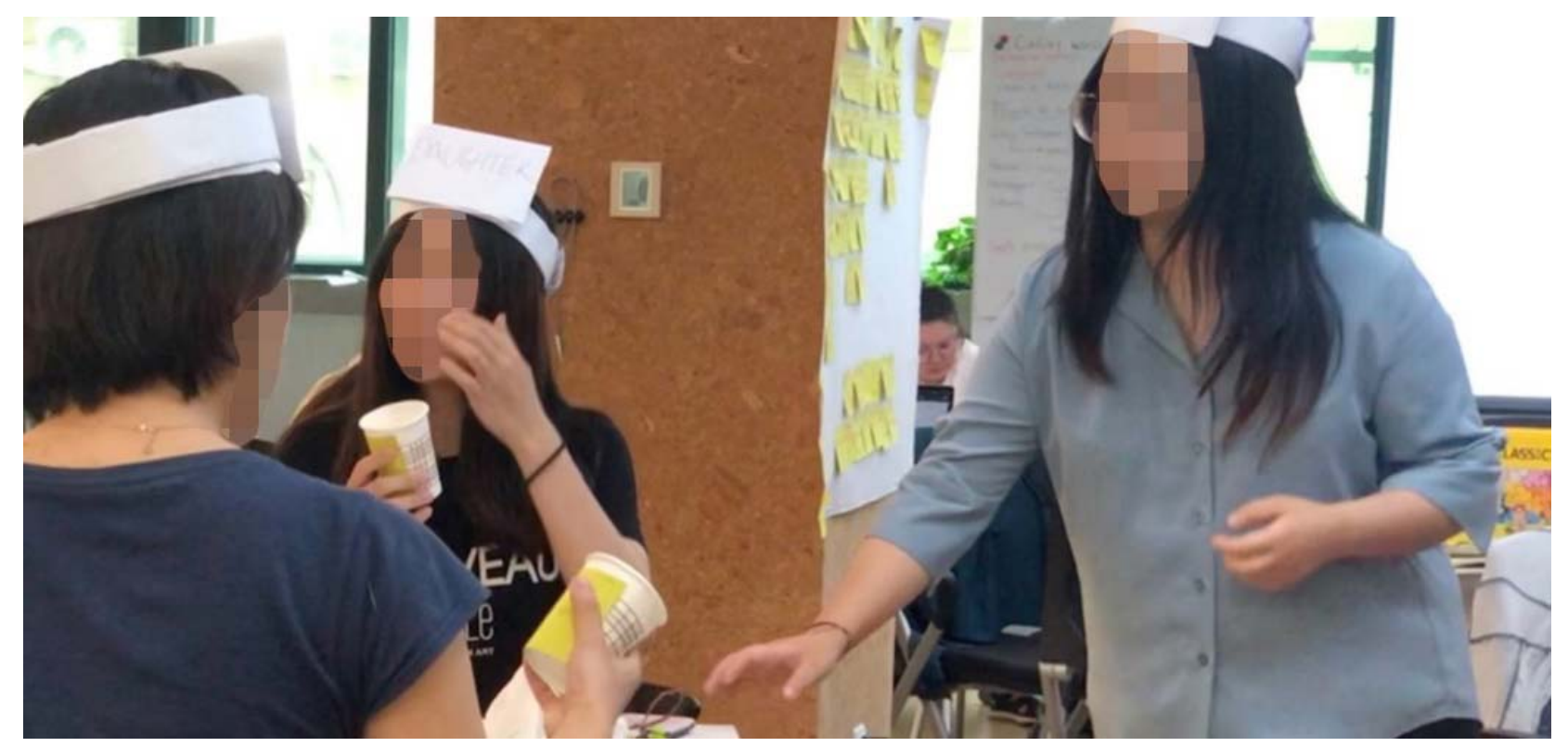

Figure 8. A scenario walkthrough of the Toast! restaurant experience.

\section{Future Perspectives}

One important principle which works on several levels is to strengthen connections: connections in the subsequent phases of a teaching and learning project, connections between AR research topics, and connections to BNUX's overall vision. For example:

- In the above AR case studies, different steps in the research were conducted, but it remained unclear how each step built on the one before it. How did the design decisions connect to the literature research and the field research? It seemed sometimes as if the literature study was done, but the design and development work only built on the field research interviews. When the final conclusion was presented, it was not connected back to the desk research. We described the process, insights, and design proposal. However, information about final implications or user validation were not included. It would be better to show how these solutions accomplished the design and research goals. There was not yet an overall vision on what the key theory, concept development, skills, and methods (or the key 'competences') were that transdisciplinary teaching and learning as an approach intends to reach. Making an overview of key competences, and then indicating in a BNUX course map how each 
course contributes to the whole, will make the story clearer and will help to develop the program further.

- The AR research topics were interesting and diverse, e.g., on a smart home and wearables. BNUX plans to identify academic and industrial communities and audiences, as well as the intended effect. After that step, it makes sense to make roadmaps for the development in an AR and VR lab. AR and VR bridge the digital and physical worlds. They allow us to take in information and content visually in the same way we take in the world. AR dramatically expands the ways the modern IoT devices can help with everyday activities like searching for information, shopping, and expressing oneself. VR lets us experience what it is like to go anywhere, from the front row of a concert to distant planets in outer space. Compared with VR, AR can put answers right where the questions are, overlaying helpful visual content and information in the real world [34].

- BNUX plans to proceed to define a 'master plan' or framework. This framework defines what 'we take on UX' is about, which competences we want to instill in the students, and what the research of the different staff members contributes toward. Such a plan helps: (1) teachers to make the pieces of courses fit together, (2) researchers to define their own research and what they can count on from their colleagues, and how to give their labs names which 'convey the unique selling point or focus area' rather than a general indication, (3) outsiders to understand 'what we really do', and what the specific UX contribution is.

- One practical action is to use the competences list and course map in a joint review at the end of each semester to summarize the teaching and learning in that semester. In this review, each teacher brings student work from the course and explains what they did in the course, and how it contributes to the competences. In this way, BNUX can grow the course map and get examples that help explain the UX and HCI framework. Similarly, this will identify in the graduation projects which student work shows off the competences we strive for best.

Author Contributions: Conceptualization, W.L.; methodology, K.-P.L.; validation, K.-H.C.; writingoriginal draft preparation, W.L.; writing—review and editing, C.M.G. and A.L.T.; supervision, L.L. All authors have read and agreed to the published version of the manuscript.

Funding: This research received no external funding.

Institutional Review Board Statement: Not applicable.

Informed Consent Statement: Informed consent was obtained from all subjects involved in the study.

Acknowledgments: We would like to thank Pieter Jan Stappers and Donald A. Norman for advising the BNUX program. We would like to thank the BNUX students for contributing to the AR, VR, and MR research projects in 2016-2021.

Conflicts of Interest: The authors declare no conflict of interest.

\section{References}

1. Yin, J.H.; Chng, C.B.; Wong, P.M.; Ho, N.; Chua, M.; Chui, C.K. VR and AR in human performance research-An NUS experience. Virtual Real. Intell. Hardw. 2020, 2, 381-393. [CrossRef]

2. Liu, W.; Byler, E.; Leifer, L. Engineering design entrepreneurship and innovation: Transdisciplinary teaching and learning in a global context. In Proceedings of the International Conference on Human-Computer Interaction; Springer: Berlin/Heidelberg, Germany, 2020; Volume 12202, pp. 451-460. [CrossRef]

3. Dym, C.; Agogino, A.; Eris, O.; Frey, D.; Leifer, L. Engineering design thinking, teaching, and learning. Eng. Educ. 2005, 94, 103-120. [CrossRef]

4. Mabogunje, A.; Sonalkar, N.; Leifer, L.; Parasker, N.; Beam, M. Regenerative learning: A process based design approach. Eng. Educ. 2020, 36, 732-748.

5. Sanders, L.; Stappers, P.J. Convivial Toolbox: Generative Research for the Front End of Design; BIS Publishers: Amsterdam, The Netherlands, 2013 .

6. Leavy, P. Essentials of Transdisciplinary Research: Using Problem-Centered Methodologies; Routledge: Abingdon, UK, 2016. 
7. Fawcett, J. Thoughts about multidisciplinary, interdisciplinary, and transdisciplinary research. Nurs. Sci. Q. 2013, 26, 376-379. [CrossRef]

8. Exter, M.E.; Gray, C.M.; Fernandez, T. Conceptions of design by transdisciplinary educators: Disciplinary background and pedagogical engagement. Technol. Des. Educ. 2020, 30, 777-798. [CrossRef]

9. Øritsland, T.A.; Buur, J. Interaction styles: An aesthetic sense of direction in interface design. Hum. Comput. Interact. 2003, 15, 67-85. [CrossRef]

10. Zimmerman, J.; Forlizzi, J.; Evenson, S. Research through design as a method for interaction design research in HCI. In Proceedings of the ACM Conference on Human Factors in Computing Systems, San Jose, CA, USA, 28 April-3 May 2007; pp. 493-502. [CrossRef]

11. Norman, D. Reflections on design. Hum. Factors Comput. Syst. 2004, 41, 1053-1054. [CrossRef]

12. Calvo, R.A.; Vella Brodrick, D.; Desmet PM, A.; Ryan, R.M. Positive computing: A new partnership between psychology, social sciences and technologists. Psychol. Well-Being Theory Res. Pract. 2016, 6, 10. [CrossRef]

13. Hekkert, P.; van Dijk, M. Vision in Design: A Guidebook for Innovators; BIS Publishers: Amsterdam, The Netherlands, 2011.

14. Faiola, A. The design enterprise: Rethinking the HCI education paradigm. Des. Issues 2007, 23, 30-45. [CrossRef]

15. Adams, R.S.; Daly, S.R.; Mann, L.M.; Dall'Alba, G. Being a professional: Three lenses into design thinking, acting, and being. Des. Stud. 2011, 32, 588-607. [CrossRef]

16. Coso Strong, A.; Lande, M.; Adams, R. Teaching without a net: Mindful design education. In Design Education Today: Technical Contexts, Programs and Best Practices; Springer: Berlin, Germany, 2019; pp. 1-21. [CrossRef]

17. Fitzgerald, D.; Ishii, H. Mediate: A spatial tangible interface for mixed reality. In Proceedings of the ACM Conference on Human Factors in Computing Systems, Montreal, QC, Canada, 21-26 April 2018; pp. 1-6. [CrossRef]

18. Liu, W.; Pasman, G.; Taal-Fokker, J.; Stappers, P.J. Exploring generation y interaction qualities at home and at work. Cogn. Technol. Work 2014, 16, 405-415. [CrossRef]

19. Lee, Y.S.; Saakes, D. Footsie: Exploring physical human-machine-interaction through flirtatious furniture. In Proceedings of the ACM Conference on Tangible, Embedded, and Embodied Interaction, Salzburg, Austria, 14-17 February 2021; pp. 1-4. [CrossRef]

20. Du, R.; Turner, E.; Dzitsiuk, M.; Prasso, L.; Duarte, I.; Dourgarian, J.; Afonso, J.; Pascoal, J.; Gladstone, J.; Cruces, N.; et al Experiencing real-time 3D interaction with depth maps for mobile augmented reality in DepthLab. In Proceedings of the ACM Symposium on User Interface Software and Technology, Virtual Event, 20-23 October 2020; pp. 108-110. [CrossRef]

21. Mayseless, N.; Saggar, M.; Hawthorne, G.; Reiss, A. Creativity in the twenty-first century: The added benefit of training and cooperation. Des. Think. Res. 2018, 239-249. [CrossRef]

22. Auernhammer, J.; Sonalkar, N.; Saggar, M. NeuroDesign: From neuroscience research to design thinking practice. Des. Think. Res. 2021, 347-355. [CrossRef]

23. Liang, C.; Lin, C.; Yao, S.; Chang, W.; Liu, Y.; Chen, S. Visual attention and association: An electroencephalography study in expert designers. Des. Stud. 2017, 48, 76-95. [CrossRef]

24. Auernhammer, J.; Liu, W.; Ohashi, T.; Leifer, L.; Byler, E.; Pan, W. NeuroDesign: Embracing neuroscience instruments to investigate human collaboration in design. In International Conference on Human Interaction and Emerging Technologies; Springer: Berlin/Heidelberg, Germany, 2020; Volume 1253, pp. 284-289. [CrossRef]

25. Saggar, M.; Volle, E.; Uddin, L.Q.; Chrysikou, E.G.; Green, A.E. Creativity and the brain: An editorial introduction to the special issue on the neuroscience of creativity. NeuroImage 2021, 231, 117836. [CrossRef] [PubMed]

26. Hecker, B.; Berger, M. Scalability of UX activities in large enterprises: An experience report from SAP AG. In International Conference on Human-Computer Interaction; Springer: Berlin/Heidelberg, Germany, 2011; Volume 6769, pp. 425-431. [CrossRef]

27. Plonka, L.; Sharp, H.; Gregory, P.; Taylor, K. UX design in agile: A DSDM case study. In Agile Software Development; Springer: Berlin/Heidelberg, Germany, 2014; Volume 179, pp. 1-15. [CrossRef]

28. Gaver, B.; Bowers, J. Annotated portfolios. ACM Interact. 2012, 19, 40-49. [CrossRef]

29. Höök, K.; Löwgren, J. Strong concepts: Intermediate-level knowledge in interaction design research. ACM Trans. Comput.-Hum. Interact. 2012, 19, 1-18. [CrossRef]

30. Kauhanen, O.; Väätäjä, H.; Turunen, M.; Keskinen, T.; Sirkkunen, E.; Uskali, T.; Lindqvist, V.; Kelling, C.; Karhu, J. Assisting immersive virtual reality development with user experience design approach. In Proceedings of the 21st International Academic Mindtrek Conference, New York, NY, USA, 20-21 September 2017; pp. 127-136. [CrossRef]

31. Zhu, Y.; Tang, G.; Liu, W.; Qi, R. How post 90's gesture interact with automobile skylight. Int. J. Hum.-Comput. Interact. 2021, 1-11. [CrossRef]

32. Greenberg, S.; Marquardt, N.; Ballendat, T.; Diaz-Marino, R.; Wang, M. Proxemic interactions-The new ubicomp? Interactions 2010, 18, 42. [CrossRef]

33. Weiser, M. The computer for the 21st Century. IEEE Pervasive Comput. 2002, 1, 19-25. [CrossRef]

34. Li, Z.; Gray, C.M.; Toombs, A.L.; McDonald, K.; Marinovic, L.; Liu, W. Cross-cultural UX pedagogy: A China-US case study. In Proceedings of the LearnxDesign: The 6th International Conference for Design Education Researchers, Jinan, China, 24-26 September 2021. 